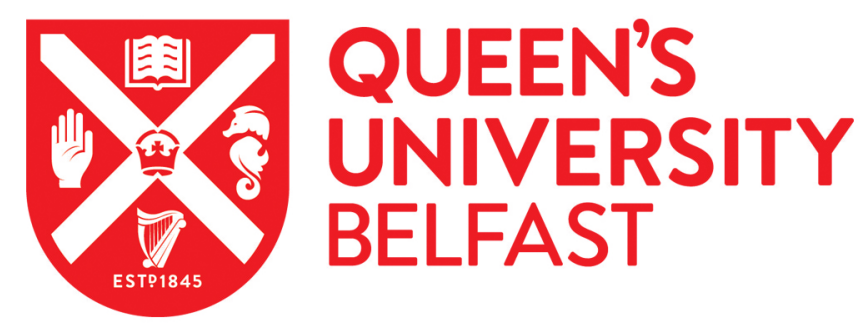

\title{
Active Fault Diagnosis via Reachable Set Separation using Interval Methods
}

Tsiakkas, M., Sopasakis, P., Boem, F., Panayiotou, C., \& Polycarpou, M. (2019). Active Fault Diagnosis via Reachable Set Separation using Interval Methods. In European Control Conference 25/06/2018 $\rightarrow$ 28/06/2019 Naples, Italy (pp. 4374-4379). Institute of Electrical and Electronics Engineers Inc.. https://doi.org/10.23919/ECC.2019.8796074

Published in:

European Control Conference 25/06/2018 $\rightarrow$ 28/06/2019 Naples, Italy

Document Version:

Peer reviewed version

Queen's University Belfast - Research Portal:

Link to publication record in Queen's University Belfast Research Portal

Publisher rights

Copyright 2019 IEEE. This work is made available online in accordance with the publisher's policies. Please refer to any applicable terms of use of the publisher.

\section{General rights}

Copyright for the publications made accessible via the Queen's University Belfast Research Portal is retained by the author(s) and / or other copyright owners and it is a condition of accessing these publications that users recognise and abide by the legal requirements associated with these rights.

Take down policy

The Research Portal is Queen's institutional repository that provides access to Queen's research output. Every effort has been made to ensure that content in the Research Portal does not infringe any person's rights, or applicable UK laws. If you discover content in the Research Portal that you believe breaches copyright or violates any law, please contact openaccess@qub.ac.uk. 


\title{
Active Fault Diagnosis via Reachable Set Separation using Interval Methods
}

\author{
Mihalis Tsiakkas ${ }^{\mathrm{a}, *}$, Pantelis Sopasakis ${ }^{\mathrm{b}}$, Francesca Boem ${ }^{\mathrm{c}}$, Christos Panayiotou ${ }^{\mathrm{a}}$ and Marios Polycarpou ${ }^{\mathrm{a}}$
}

\begin{abstract}
This paper presents an optimization-based approach for active fault isolation in linear time invariant systems subject to additive disturbances which are bounded in intervals. We model the evolution of the system states and outputs using interval dynamics with the input signals also bounded within given intervals. The proposed approach is based on determining a sequence of control actions to minimize a function which quantifies the overlap of output intervals, while taking into account the prescribed input bounds. The resulting optimization problem is nonconvex, yet the cost function has Lipschitzcontinuous gradient and the constraints are simple, which allows us to solve them efficiently. A numerical example is used to illustrate the implementation and suitability of the proposed optimization approach for active fault isolation.
\end{abstract}

\section{INTRODUCTION}

Fault diagnosis methods can be separated into two broad classes [1]: the first group of methods includes passive fault diagnosis techniques, where input/output data is used to make a decision about the health status of the system. In the second group, represented by so-called active methods (see [2] for a recent survey), the control input is optimized to help diagnose a fault; these methods are termed active fault diagnosis (AFD). A major shortcoming of traditional passive fault diagnosis techniques is that feedback controllers can compensate the effect of faults, thus hiding their presence or making them difficult to be distinguished. This may result in undesirable delays in in detecting and/or isolating faults, possibly leading to more severe consequences or propagation to major failures in the system.

As explained in [2], many different methods and scenarios have been proposed under the umbrella of AFD, considering deterministic [3], [4] or stochastic uncertainties [5], [6], hybrid stochastic-deterministic approaches [7], [8], and finite or infinite AFD auxiliary control sequences [9].

The proposed approach belongs to the class of deterministic methods for model discrimination. Recent works have investigated the use of set membership methods, analyzing the reachable sets of the nominal and a bank of faulty models [10]. A common approach is based on the use of zonotopes [11] to describe the bounded uncertainties models,

\footnotetext{
a KIOS Research and Innovation Center of Excellence and the Department of Electrical and Computer Engineering, University of Cyprus, Nicosia, Cyprus.

b Queen's University Belfast, School of EEECS, i-AMS Centre.

c Department of Electronic and Electrical Engineering, University College London, London, UK.

This work has been supported by European Union's Horizon 2020 research and innovation programme (KIOS CoE) under Grant 739551.

* Corresponding author. Email: m.tsiakkas e ieee.org
}

both for fault detection [12] and diagnosis [13], [14]. A similar approach is pursued here using interval methods which simplify the computation of model-to-model distances.

As pointed out in [2], the implementation of active fault diagnosis techniques in practical settings is still limited, mainly due to the high computational complexity that these methods require. Even though some approaches have been suggested to alleviate the computational complexity (see for example [15]), this drawback hinders the applicability of AFD to even low-dimensional systems or networks of few interconnected systems [16]. This is mainly due to the fact that the design of the separating input is performed via the solution of a mixed integer quadratic program, which is not suitable for real-time implementations.

In this paper, we propose a novel formulation of the active fault diagnosis problem which does not require the use of mixed-integer formulations. More specifically, we present a set membership approach towards active fault isolation utilizing interval methods. Similar to previous works [10], we assume each fault is described by a known a priori fault model subject to deterministically bounded uncertainties which are modeled by an interval uncertainty vector.

We propose an optimization-based approach, which aims to derive a finite sequence of control actions that minimizes the overlapping of possible model outputs. In order to avoid formulating mixed-integer problems, we introduce an appropriate inexact penalty function which is sufficiently smooth and allows the use of efficient numerical algorithms. The resulting optimization problem turns out to be a smooth nonconvex problem with simple constraints which can be solved efficiently.

Summing up, the main contributions of this paper are:

1) An active fault isolation method for systems with interval uncertainties, not requiring the solution of mixed integer problems, neither online nor offline;

2) An optimization-based approach which attempts the simultaneous separation of multiple fault models by minimizing the overlapping. This leads to the formulation of a nonconvex problem which possesses a favorable structure, allowing the use of fast methods for its solution;

3) We show the effectiveness of the proposed methodology with an example regarding a ground robot.

The rest of the paper is structured as follows. The adopted notation and some useful preliminary results are presented in Section II. In Section III the considered problem is illustrated and the use of intervals for system dynamics representation is introduced in Section IV. The proposed approach for active 
fault isolation is then presented in Section $\mathrm{V}$. Numerical simulations are shown in Section $\mathrm{VI}$ and some concluding remarks can be found in Section VII

\section{PRELIMINARIES}

Hereafter, we denote by $\mathbb{R}, \mathbb{R}^{n}, \mathbb{R}^{m \times n}, \mathbb{N}$ and $\mathbb{N}_{\left[k_{1}, k_{2}\right]}$ the sets of real numbers, $n$-dimensional real vectors, $m$-by- $n$ real matrices, nonnegative integers and the set of nonnegative integers which are in the interval $\left[k_{1}, k_{2}\right]$. We denote the transpose of a matrix $A$ by $A^{\top}$.

The interval $\boldsymbol{x}=[\underline{x}, \bar{x}]$ where $\underline{x}, \bar{x} \in \mathcal{X} \subseteq \mathbb{R} \cup\{\infty\}$ and $\underline{x} \leq \bar{x}$ represents the range of numbers $\{x \in \mathcal{X} \mid \underline{x} \leq$ $x \leq \bar{x}\}$. For the sake of clarity, we, henceforth, denote all intervals by boldface letters. The field of intervals over a set $\mathcal{X} \subseteq \mathbb{R}$ is denoted by $[\mathcal{X}]$. This notation is extended to interval vectors in $\mathcal{X} \subseteq \mathbb{R}^{n}$ where $\leq$ is meant in the element-wise sense.

For intervals $\boldsymbol{x}=[\underline{x}, \bar{x}] \in\left[\mathbb{R}^{n}\right]$ and $\boldsymbol{y}=[y, \bar{y}] \in\left[\mathbb{R}^{m}\right]$ we define their Cartesian product, $\boldsymbol{x} \times \boldsymbol{y}$, to be the interval $\boldsymbol{x} \times \boldsymbol{y}=\left[\left[\underline{x}^{\top} \underline{y}^{\top}\right]^{\top},\left[\bar{x}^{\top} \bar{y}^{\top}\right]^{\top}\right] \in\left[\mathbb{R}^{n+m}\right]$.

Before proceeding, we define the following interval operations. Let $\boldsymbol{x}=[\underline{x}, \bar{x}] \in[\mathbb{R}]$ and $\boldsymbol{y}=[y, \bar{y}] \in[\mathbb{R}]$. Then $\boldsymbol{x}+\boldsymbol{y}=[\underline{x}+\underline{y}, \bar{x}+\bar{y}]$ and $\boldsymbol{x}-\boldsymbol{y}=[\underline{x}-\bar{y}, \bar{x}-\underline{y}]$. Furthermore, the width of an interval is given by $|\boldsymbol{x}|_{[\mathbb{R}]}:=\bar{x}-\underline{x}$, while its center point is given by $c(\boldsymbol{x})=\frac{1}{2}(\bar{x}+\underline{x})$. The center and width operators are extended to $\left[\mathbb{R}^{n}\right]$ in the elementwise sense. Vectors $t \in \mathbb{R}^{n}$ can be, trivially, identified by intervals $\boldsymbol{t} \in\left[\mathbb{R}^{n}\right]$ with $c(\boldsymbol{t})=t$ and $|\boldsymbol{t}|_{[\mathbb{R}]}=0$.

The following lemma is provided without proof but follows easily from basic interval arithmetic operations

Lemma 1: Let $\boldsymbol{x}, \boldsymbol{y} \in[\mathbb{R}]$ and $\alpha, \beta \in \mathbb{R}$, then

$$
\begin{gathered}
|\alpha \boldsymbol{x}+\beta \boldsymbol{y}|_{[\mathbb{R}]}=|\alpha||\boldsymbol{x}|_{[\mathbb{R}]}+|\beta||\boldsymbol{y}|_{[\mathbb{R}]} \\
\text { and } c(\alpha \boldsymbol{x}+\beta \boldsymbol{y})=\alpha c(\boldsymbol{x})+\beta c(\boldsymbol{y}) .
\end{gathered}
$$

As a result of the above lemma we have that given $A \in \mathbb{R}^{m \times n}$ and $\boldsymbol{x} \in\left[\mathbb{R}^{n}\right]$ then $c(A \boldsymbol{x})=A c(\boldsymbol{x})$ and $|A \boldsymbol{x}|_{[\mathbb{R}]}=|A||\boldsymbol{x}|_{[\mathbb{R}]}$ where $|A|=\left[\left|a_{i j}\right|\right]_{i, j}$. The above lemma suggests that $|\cdot|_{[\mathbb{R}]}$ is an additive seminorm and $c(\cdot)$ is a linear operator.

A discrete-time, linear, time-invariant (LTI) system is a dynamical system which is identified by the tuple $P=$ $(A, B, C, D)$ with dynamics

$$
\begin{aligned}
x(k+1) & =A x(k)+B u(k)+v(k), \\
y(k) & =C x(k)+D u(k)+w(k),
\end{aligned}
$$

where $x \in \mathbb{R}^{n_{x}}$ is the system state, $u \in \mathbb{R}^{n_{u}}$ is the manipulated input, $y \in \mathbb{R}^{n_{y}}$ is the measured output, while $v \in \mathbb{R}^{n_{x}}$ and $w \in \mathbb{R}^{n_{y}}$ are disturbances acting on the system dynamics and output respectively. Using the above definitions for interval arithmetic, LTI systems can be extended to accommodate interval-valued states $\boldsymbol{x}(k)=[\underline{x}(k) \bar{x}(k)]^{\top} \in$ $\left[\mathbb{R}^{n_{x}}\right]$ and interval disturbances $\boldsymbol{v} \in\left[\mathbb{R}^{n_{x}}\right]$ and $\boldsymbol{w} \in\left[\mathbb{R}^{n_{y}}\right]$. Throughout this paper, it is assumed that disturbances and uncertainties are bounded and that the corresponding bounds are time-invariant, hence $\boldsymbol{v}$ and $\boldsymbol{w}$ are independent of $k$.

\section{PROBLEM Formulation}

Suppose that the system we want to monitor can be described by a nominal model $P_{0}$ and $N$ known possible faulty models, each of which is described by an LTI system of the same order as $P_{0}$. Now, let each of the faulty models be denoted $P_{i}$ for $i \in \mathbb{N}_{[1, N]}$ and have dynamics given by (2) where $P_{i}=\left(A_{i}, B_{i}, C_{i}, D_{i}\right)$. Moreover, $\boldsymbol{x}_{i}(k)=$ $\left[\underline{x}_{i}(k), \bar{x}_{i}(k)\right] \in\left[\mathbb{R}^{n_{x}}\right]$ is the interval state vector and $\boldsymbol{v}_{i}=$ $\left[\underline{v}_{i}, \bar{v}_{i}\right] \in\left[\mathbb{R}^{n_{x}}\right]$ and $\boldsymbol{w}_{i}=\left[\underline{w}_{i}, \bar{w}_{i}\right] \in\left[\mathbb{R}^{n_{y}}\right]$ are the state and output interval uncertainty vectors respectively. Finally, $u(k) \in \mathbb{R}^{n_{u}}$ is the system manipulated input.

Suppose that at time $k_{f}$ a fault is detected in the system. It is important to identify and diagnose the source of the fault which would then allow for the restructuring of the control system in order to maintain stability and safe operation. This step can be achieved using active fault isolation techniques; an interval based approach to this purpose is presented in this paper. Since the fault detection module might produce false alarms, the nominal model $P_{0}$ will also be considered as a possible contingency for model isolation.

The objective of this work is to design an input sequence $U=\left\{u\left(k_{f}\right), \ldots, u\left(k_{f}+k_{h}\right)\right\}$ over a horizon $k_{h}$ such that the output interval vectors do not overlap (see Fig. 11 for an illustrative explanation), thus allowing for the diagnosis and possible isolation of the system fault. This way, by applying the computed input sequence, the measurements will be consistent with a single fault model only. Interval arithmetic is used to model both the nominal and faulty cases so as to capture bounded uncertainties and non-linearities in the physical system.

A brief motivating example is given below. Consider the LTI model

$P_{i}=\left(\left[\begin{array}{cc}-0.083 & -0.051 \\ 0.051 & -0.083\end{array}\right],\left[\begin{array}{cc}-0.102 & i-0.319 \\ 0.5 i & 0.313\end{array}\right],\left[\begin{array}{cc}-0.865 & -0.030 \\ 0.929 & 0.350\end{array}\right], 0\right)$

with $i \in \mathbb{N}_{[0,1]}$ and disturbances $\boldsymbol{v}_{i}$ and $\boldsymbol{w}_{i}$ satisfying $c\left(\boldsymbol{v}_{i}\right)=c\left(\boldsymbol{w}_{i}\right)=0$ and $\left|\boldsymbol{v}_{1}\right|_{[\mathbb{R}]}^{\top}=\left[\begin{array}{ll}0.376 & 0.128\end{array}\right],\left|\boldsymbol{v}_{2}\right|_{[\mathbb{R}]}^{\top}=$ $\left[\begin{array}{ll}0.2530 .349\end{array}\right], \quad\left|\boldsymbol{w}_{1}\right|_{[\mathbb{R}]}^{\top}=\left[\begin{array}{lll}0.420 & 0.197\end{array}\right]$ and $\left|\boldsymbol{w}_{2}\right|_{[\mathbb{R}]}^{\top}=$ [ 0.1270 .251$]$. Now suppose that at time $k=k_{f}$ a fault is detected and furthermore suppose that (for simplicity) $x_{i}\left(k_{f}\right)=0$. Figures 1 and 2 show the responses of the two models to the input sequences

$$
U^{\mathrm{s}}=\left\{\left[\begin{array}{l}
-0.021 \\
-0.062
\end{array}\right],\left[\begin{array}{l}
0.013 \\
0.765
\end{array}\right]\right\}, U^{\mathrm{ns}}=\left\{\left[\begin{array}{l}
0.376 \\
0.128
\end{array}\right],\left[\begin{array}{l}
0.253 \\
0.349
\end{array}\right]\right\},
$$

respectively. It can be seen by the two figures that under $U^{\mathrm{s}}$ the output intervals of the two models are completely separated (i.e., they do not intersect), hence an output measurement at time $k=k_{f}+2$ would allow one to deduce which of the two models is active thus diagnosing the fault. On the other hand, with $U^{\text {ns }}$ the intersection between the two output interval vectors means that the possibility exists that the output measurement can be explained by both models.

\section{INTERVAL EVOLUTION MODELING}

In this section we study the modeling aspects of dynamical systems whose states, output and disturbance signals are interval valued. We focus on the structure given by with 


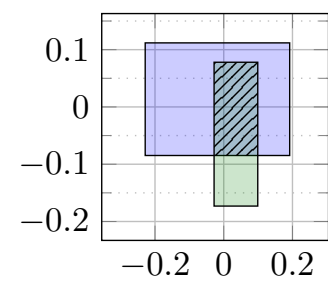

(a) $k=k_{f}+1$.

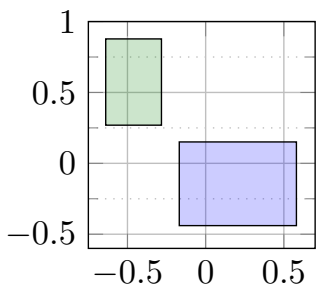

(b) $k=k_{f}+2$
Fig. 1: Response to separating input sequence $U^{\mathrm{s}}$.

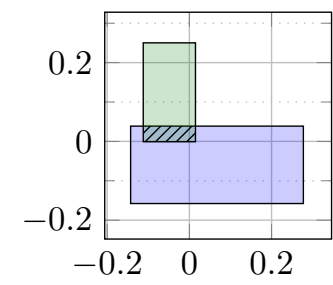

(a) $k=k_{f}+1$.

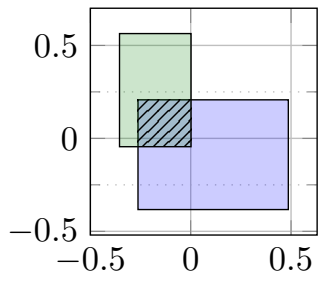

(b) $k=k_{f}+2$

Fig. 2: Response to non-separating input sequence $U^{\text {ns }}$.

the various variables as defined in the problem formulation. It turns out that the centers and widths of the state and output interval vectors follow an LTI dynamics resulting in a state space parametrization with double the number of states in relation to the original model.

By applying Lemma 1 to $2 \mathrm{a}$ we obtain the following dynamics for the width of the state vector, $\tilde{x}_{i}(k):=\left|\boldsymbol{x}_{i}(k)\right|_{[\mathbb{R}]}$,

$$
\begin{aligned}
\tilde{x}_{i}(k+1) & =\left|A_{i} \boldsymbol{x}_{i}(k)+B_{i} u(k)+\boldsymbol{v}_{i}\right|_{[\mathbb{R}]} \\
& =\left|A_{i} \boldsymbol{x}_{i}(k)\right|_{[\mathbb{R}]}+\left|B_{i} u(k)\right|_{[\mathbb{R}]}+\left|\boldsymbol{v}_{i}\right|_{[\mathbb{R}]} \\
& =\left|A_{i}\right|\left|\boldsymbol{x}_{i}(k)\right|_{[\mathbb{R}]}+\left|\boldsymbol{v}_{i}\right|_{[\mathbb{R}]}
\end{aligned}
$$

where $\left|B_{i} u(k)\right|_{[\mathbb{R}]}=0$ since $B_{i} u(k) \in \mathbb{R}^{n}$ is a vector (due to the fact that $u(k)$ is assumed to be perfectly known) which, when interpreted as an interval, has zero width. Therefore,

$$
\tilde{x}_{i}(k+1)=\left|A_{i}\right| \tilde{x}_{i}(k)+\left|\boldsymbol{v}_{i}\right|_{[\mathbb{R}]},
$$

which yields

$$
\tilde{x}_{i}(k)=\left|A_{i}\right|^{k} \tilde{x}_{i}(0)+\sum_{i=0}^{k-1}\left|A_{i}\right|^{i}\left|\boldsymbol{v}_{i}\right|_{[\mathbb{R}]} .
$$

Since all elements of $\left|A_{i}\right|$ and $\left|\boldsymbol{v}_{i}\right|_{[\mathbb{R}]}$ are non-negative, it follows that $\tilde{x}_{i}(k+1) \geq \tilde{x}_{i}(k)$ for all $k \geq 1$.

Similarly, using (1) the dynamics of the center of $x_{i}(k)$, denoted $\check{x}_{i}(k):=c\left(x_{i}(k)\right)$, is given by

$$
\begin{aligned}
c\left(\boldsymbol{x}_{i}(k+1)\right) & =c\left(A_{i} \boldsymbol{x}_{i}(k)+B_{i} u(k)+\boldsymbol{v}_{i}\right) \\
& =A_{i} c\left(\boldsymbol{x}_{i}(k)\right)+B_{i} u(k)+c\left(\boldsymbol{v}_{i}\right) .
\end{aligned}
$$

Hence,

$$
\check{x}_{i}(k+1)=A_{i} \check{x}_{i}(k)+B_{i} u(k)+c\left(\boldsymbol{v}_{i}\right) .
$$

Finally, combining the above we have the dynamics of the interval-valued state $\boldsymbol{x}$ is given in terms of its width and center as follows

$$
\left[\begin{array}{c}
\tilde{x}_{i}(k+1) \\
\check{x}_{i}(k+1)
\end{array}\right]=\left[\begin{array}{ll}
\left|A_{i}\right| & \\
& A_{i}
\end{array}\right]\left[\begin{array}{c}
\tilde{x}_{i}(k) \\
\check{x}_{i}(k)
\end{array}\right]+\left[\begin{array}{c}
0 \\
B_{i}
\end{array}\right] u(k)+\left[\begin{array}{c}
\left|\boldsymbol{v}_{i}\right|_{[\mathbb{R}]} \\
c\left(\boldsymbol{v}_{i}\right)
\end{array}\right] .
$$

Similarly, let $\check{y}_{i}(k)$ and $\tilde{y}_{i}(k)$ denote the center and width of the output vector at time $k$ respectively (i.e., $\check{y}_{i}(k):=$ $c\left(\boldsymbol{y}_{i}(k)\right)$ and $\left.\tilde{y}_{i}(k):=\left|\boldsymbol{y}_{i}(k)\right|_{[\mathbb{R}]}\right)$. Then

$$
\left[\begin{array}{c}
\tilde{y}_{i}(k) \\
\check{y}_{i}(k)
\end{array}\right]=\left[\begin{array}{ll}
\left|C_{i}\right| & \\
& C_{i}
\end{array}\right]\left[\begin{array}{c}
\tilde{x}_{i}(k) \\
\check{x}_{i}(k)
\end{array}\right]+\left[\begin{array}{c}
0 \\
D_{i}
\end{array}\right] u(k)+\left[\begin{array}{c}
\left|\boldsymbol{w}_{i}\right|_{[\mathbb{R}]} \\
c\left(\boldsymbol{w}_{i}\right)
\end{array}\right] .
$$

Using (4), the state interval vector center $\check{x}_{i}(k)$ can be expressed in terms of $u(k)$ as follows

$$
\check{x}_{i}(k)=A_{i}^{k} \check{x}_{i}(0)+\sum_{t=0}^{k-1} A_{i}^{k-1-t} B_{i} u(t)+\sum_{t=0}^{k-1} A_{i}^{t} c\left(\boldsymbol{v}_{i}\right)
$$

By defining $\Gamma(A, B, k)$ as

$$
\Gamma(A, B, k)=\left[\begin{array}{llll}
A^{k-1} B & \cdots & A B & B
\end{array}\right],
$$

the expression for $\check{x}_{i}(k)$ given by (6) can be simplified to

$$
\begin{aligned}
& \check{x}_{i}(k)=A_{i}^{k} \check{x}_{i}(0)+\sum_{t=0}^{k-1} A_{i}^{t} c\left(\boldsymbol{v}_{i}\right) \\
& +\left[\Gamma\left(A_{i}, B_{i}, k\right) 0\right] U(k)
\end{aligned}
$$

where $U(k)=\left[\begin{array}{lll}u(0)^{\top} & \cdots & u(k)^{\top}\end{array}\right]^{\top}$.

Now substituting (3) and (7) into (5) we obtain

$$
\begin{aligned}
& \tilde{y}_{i}(k)=\left|C_{i}\right|\left|A_{i}\right|^{k} \tilde{x}_{i}(0)+\left|C_{i}\right| \sum_{t=0}^{k-1}\left|A_{i}\right|^{t}\left|\boldsymbol{v}_{i}\right|_{[\mathbb{R}]}+\left|\boldsymbol{w}_{i}\right|_{[\mathbb{R}]}, \\
& \check{y}_{i}(k)=G_{i}(k)+\left[C_{i} \Gamma\left(A_{i}, B_{i}, k\right) \quad D_{i}\right] U(k),
\end{aligned}
$$

where

$$
G_{i}(k)=C_{i} A_{i}^{k} \check{x}_{i, 0}+C_{i} \sum_{l=0}^{k-1} A_{i}^{l} c\left(\boldsymbol{v}_{i}\right)+c\left(\boldsymbol{w}_{i}\right) .
$$

Then define an output function $\delta_{i, j}(k)$, which depends also on $U$, for models $i$ and $j$ whose $l^{\text {th }}$ element is given by

$$
\delta_{i, j}^{l}(k)=\left(\xi_{i, j}^{l}(k)\right)^{2}-\left(\vartheta_{i, j}^{l}(k)\right)^{2},
$$

where

$$
\begin{gathered}
\xi_{i, j}(k)=\left|\check{y}_{i}(k)-\check{y}_{j}(k)\right| \text { and } \\
\vartheta_{i, j}(k)=\frac{1}{2}\left(\tilde{y}_{i}(k)+\tilde{y}_{j}(k)\right),
\end{gathered}
$$

the elements of which quantify the distance between the state intervals, $\boldsymbol{x}_{i}(k)$ and $\boldsymbol{x}_{j}(k)$, of the two models along each direction.

\section{Active Interval SeParation}

We now proceed to the main results of this paper. In this section, some separation conditions are given first followed by an optimization-based approach where an attempt is made to simultaneously separate all models. 


\section{A. Separation conditions}

We begin by formally defining the terms separation and separability in the context of interval arithmetic. Let $\boldsymbol{x} \in$ $\left[\mathbb{R}^{n}\right]$ and $\boldsymbol{y} \in\left[\mathbb{R}^{n}\right]$. The two intervals vectors are said to be separated if $\boldsymbol{x} \cap \boldsymbol{y}=\emptyset$. Now suppose that the interval vectors being considered are parametrized by $\theta \in \Theta$; then the two are said to be separable if there exists a $\theta \in \Theta$ such that $\boldsymbol{x}(\theta) \cap \boldsymbol{y}(\theta)=\emptyset$.

Lemma 2: Two intervals $\boldsymbol{x}, \boldsymbol{y} \in\left[\mathbb{R}^{n}\right]$ are separated if and only if there exists an $l \in \mathbb{N}_{[1, n]}$ so that $\boldsymbol{x}^{l}$ and $\boldsymbol{y}^{l}$ are separated where $\boldsymbol{x}^{l}$ and $\boldsymbol{y}^{l}$ denote the $l^{\text {th }}$ elements of $\boldsymbol{x}$ and $\boldsymbol{y}$ respectively.

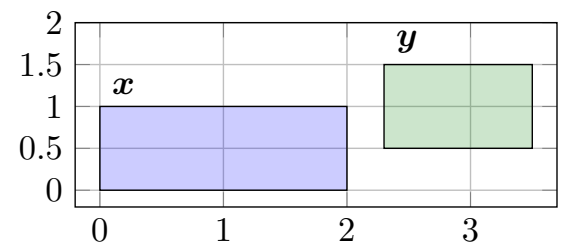

Fig. 3: Graphical proof of Lemma 2

Equipped with the above lemma, we say that two interval vectors $\boldsymbol{x}, \boldsymbol{y} \in\left[\mathbb{R}^{n}\right]$ are separated along direction $l \in \mathbb{N}_{[1, n]}$ if $\boldsymbol{x}^{l} \cap \boldsymbol{y}^{l}=\emptyset$.

The separation of two intervals can be established based on their centres and widths as outlined in the following lemma.

Lemma 3: Two intervals $\boldsymbol{x}, \boldsymbol{y} \in[\mathbb{R}]$ are separated if and only if $|c(\boldsymbol{x})-c(\boldsymbol{y})|>\frac{1}{2}\left(|\boldsymbol{x}|_{[\mathbb{R}]}+|\boldsymbol{y}|_{[\mathbb{R}]}\right)$.

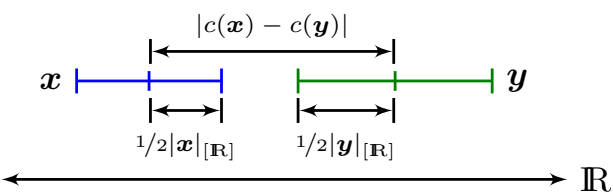

Fig. 4: Graphical proof of Lemma 3

Figure 3 gives a graphical proof for Lemma 2, where it can be seen that the two intervals $\boldsymbol{x}$ and $\boldsymbol{y}$ are overall separated even though they overlap in the vertical direction. Similarly, a proof of Lemma 3 above is given in Figure 4

The following theorem exploits the measure defined in (8) in conjunction with the results presented in Lemmas 2 and 3 above, to test whether the output interval vectors of two models are separated.

Theorem 4 (Separation criterion): Consider two models $P_{i}$ and $P_{j}$ of the form given in (2) extended as in (4) and (5) and let $\delta_{i, j}(k)$ be defined as in $(8)$. Then the output interval vectors of the two models are separated at time $k$ if and only if at least one element of $\delta_{i, j}(k)$ is positive.

Proof. The result follows by applying Lemmas 2 and 3

\section{B. Optimization-based simultaneous separation}

Suppose that as before we have $N+1$ models 1 for which we wish to separate the reachable state intervals.

\footnotetext{
${ }^{1}$ The nominal model plus $N$ possible faults.
}

Additionally, as it is typically the case in practice, assume that the input variables are constrained to be within an interval $\mathcal{U} \in\left[\mathbb{R}^{n_{u}}\right]$. Using $\delta_{i, j}(k)$ as defined in (8) and following Theorem 4, a necessary and sufficient condition for separation to be achieved at time index $k$ is that

$$
\min \left(-\delta_{i, j}(k)\right)<0,
$$

for all $i, j \in \mathbb{N}_{[0, N]}$ with $i \neq j$.

We define the operator $[\cdot]_{+}: \mathbb{R} \rightarrow \mathbb{R}_{+}$as follows

$$
[x]_{+}:= \begin{cases}0 & \text { if } x \leq 0 \\ x & \text { if } x>0\end{cases}
$$

Now let $\epsilon>0$ be a positive constant and denote by $\delta_{i, j}^{l}(k)$ the $l^{\text {th }}$ element of $\delta_{i, j}(k)$. Then (9) is equivalent to

$$
\phi_{i, j}(U, k)=0,
$$

for $i, j \in \mathbb{N}_{[0, N]}$, where $\phi_{i, j}: \mathbb{R}^{n_{u} k} \times \mathbb{N} \rightarrow \mathbb{R}_{+}$is the function

$$
\phi_{i, j}(U, k):=\prod_{l=1}^{n_{y}}\left[\epsilon-\delta_{i, j}^{l}(k)\right]_{+}^{2} .
$$

We may also define the following measure of overlap at time $k$ as

$$
\Phi(U, k):=\sum_{\substack{i, j=0 \\ i \neq j}}^{N} \phi_{i, j}(U, k) .
$$

In order for all $N$ subsystems to be separated at time $k$, it is desirable that $\Phi(U, k)$ becomes sufficiently small as explained in the following proposition.

Lemma 5: Assume that there exists a separating sequence in $\mathfrak{U}_{k-1}$ for some $k \in \mathbb{N}$. Let $U$ satisfy $\Phi(U, k)<\epsilon^{2 n_{y}}$. Then, $U$ is a separating sequence.

Proof. For a sequence $x_{1}, \ldots, x_{n} \geq 0$. with $\prod_{i=1}^{n} x_{i} \leq \epsilon^{n}$, it holds that $x_{i} \leq \epsilon$ for some $i$. Indeed, if not, $x_{i}>\epsilon$ for all $i$ and $\prod_{i=1}^{n} x_{i}>\epsilon^{n}-$ a contradiction.

Since $\Phi(U, k)<\epsilon^{2 n_{y}}$, we have that $\phi_{i, j}(U, k)<\epsilon^{2 n_{y}}$ for every pair $i, j \in \mathbb{N}_{[0, N]}, i \neq j$. In turn, this implies that there is an $l \in \mathbb{N}_{\left[1, n_{y}\right]}$ so that $\left[\epsilon-\delta_{i, j}^{l}(k)\right]_{+}<\epsilon$, therefore, $\delta_{i, j}^{l}(k)>0$, which completes the proof.

Lemma 5 provides a criterion for separation at time $k$ using $\Phi(U, k)$ as a gauge function. In fact, we are interested in achieving separation at any time $k$ no larger than a given maximum time $k_{\max }$. To that end, we define

$$
\Psi_{\delta}\left(U, k_{\max }\right):=\prod_{k=1}^{k_{\max }} \Phi\left(U_{[k]}, k\right),
$$

where $U_{[k]}=\left[\begin{array}{lll}u(0)^{\top} \ldots u(k)^{\top} & \ldots\end{array}\right]^{\top}$ and $U=U_{\left[k_{\max }\right]}$.

We will show that the models are separated at some $k_{0} \in$ $\mathbb{N}_{\left[1, k_{\max }\right]}$ if $\Psi_{\delta}\left(U, k_{\max }\right)$ becomes sufficiently small.

Our main goal is to determine a solution $U$ to $\Psi_{\delta}\left(U, k_{\max }\right)=0$, however, we are interested in doing so while meeting two more requirements. 
The first is that, especially for open-loop unstable systems, it is desirable that the system state remains as close as possible to a given reference value, $r(k)$. To enforce that the state of all subsystems does not stray too much from the prescribed set point, we may use the cost function

$$
\Psi_{r}\left(U, k_{\max }\right)=\sum_{k=1}^{k_{\max }} \sum_{i=0}^{N}\left\|Q\left(\check{y}_{i}(k)-r(k)\right)\right\|_{2}^{2},
$$

where $Q \in \mathbb{R}^{n_{y} \times n_{y}}$ is a weighting matrix.

The second objective is that we need to achieve separation at a minimum actuation effort. We naturally introduce the following actuation cost function

$$
\Psi_{u}(U)=\|R U\|_{2}^{2}
$$

for some weighting matrix $R \in \mathbb{R}^{n_{u}\left(k_{\max }+1\right) \times n_{u}\left(k_{\max }+1\right)}$.

We define the following optimization problem

$$
\mathcal{P}(\lambda): \operatorname{minimize}_{U \in \mathfrak{U}_{k_{\max }-1}} \lambda \Psi_{\delta}\left(U, k_{\max }\right)+\Psi_{r}\left(U, k_{\max }\right)+\Psi_{u}(U)
$$

where $\mathfrak{U}_{k}=\mathcal{U} \times \cdots \times \mathcal{U}$ ( $k$ times) and $\times$ is a Cartesian product of intervals defined in Section III

Problem $\mathcal{P}(\lambda)$ is a nonconvex problem which has a $C^{1}$ cost with locally Lipschitz gradient and constraints on which we may easily compute projections (either analytically or numerically). Additionally, in all problems of practical interest, primarily for safety reasons, sets $\mathcal{U}$ are bounded. Under these assumptions, there exist very efficient algorithms, which are suitable for embedded applications using which we may solve such problems [17], [18].

With this optimization problem, if feasible, we find a minimum-norm actuation sequence which leads to high separation at an early stage.

In order to enforce that $\Psi_{\delta}\left(U, k_{\max }\right)=0$, we employ the penalty method [19], [20] which boils down to the iterative procedure of Algorithm 1 .

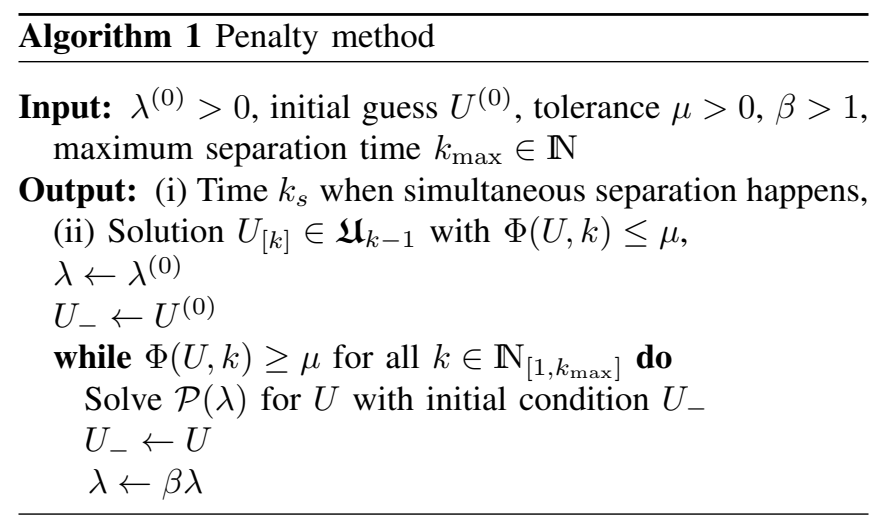

Lemma 6 (Convergence of Algorithm 1): Assume that there exists a separating sequence in $\mathfrak{U}_{k-1}$ for some $k \in$ $\mathbb{N}_{\left[1, k_{\max }\right]}$ and let $U^{\nu}$ be a solution of $\mathcal{P}\left(\lambda^{\nu}\right)$ and $\lambda^{\nu} \rightarrow \infty$. Then, all cluster points, $U^{\star}$ of $U^{\nu}$ satisfy $\Psi_{\delta}\left(U^{\star}, k_{\max }\right)=0$ and the conditions of Lemma 5 .

Proof. Define $f^{\nu}(U)=\lambda^{\nu} \Psi_{\delta}\left(U, k_{\max }\right)+\Psi_{r}\left(U, k_{\max }\right)+$ $\Psi_{u}(U)$. Then, the sequence $f^{\nu}$ epi-converges to $f(U)=$
$g(U)+\Psi_{r}\left(U, k_{\max }\right)+\Psi_{u}(U)$, where

$$
g(U)= \begin{cases}0, & \text { if } \Psi_{\delta}\left(U, k_{\max }\right)=0 \\ +\infty, & \text { otherwise }\end{cases}
$$

as it can be easily seen by [21, Def. 7.1, Ex. 4.3b]. According to [21, Thm. 7.33] (all requirements of which are satisfied), $\inf _{U} f^{\nu}(U) \rightarrow \inf _{U} f(U)$ and $\limsup \left(\arg \min f^{\nu}\right) \subseteq$ $\arg \min f$, where $\limsup$ here denotes the outer limit [21, Def. 4.1]. Since $U^{\nu} \in \arg \min f^{\nu}$, every cluster point $U^{\star}$ of $\left(U^{\nu}\right)_{\nu}$ is a minimizer of $f$, thus satisfies $\Psi_{\delta}\left(U^{\star}, k_{\max }\right)=0$, which proves the assertion.

Note that since $\mathfrak{U}_{k_{\max }-1}$ is bounded, $U^{\nu}$ does have a cluster point by the Bolzano-Weierstrass Theorem [22, Sec 11.2]. For the same reason, $\lim \sup \left(\arg \min f^{\nu}\right)$ is non-empty.

As a result of Lemma 6, some iterate $U$ of Algorithm 1 will satisfy $\Psi_{\delta}\left(U, k_{\max }\right)<\epsilon^{2 n_{y} k_{\max }}$. This implies that there is a $k \in \mathbb{N}_{\left[1, k_{\max }\right]}$ so that $\Phi(U, k)<\epsilon^{2 n_{y}}$, so by virtue of Lemma 5, we obtain a separating sequence.

Remark 7: In any practical scenario, faster separation is preferable. To that end, the following extension to our cost function can be employed.

$$
\Psi_{t}\left(U, k_{\max }\right)=\sum_{k=1}^{k_{\max }} 2^{-\rho k} \Phi\left(U_{[k]}, k\right),
$$

for some coefficient $\rho>0$. This function penalizes lower values of $k$ thus encouraging the solver to produce a shorter separating input sequence.

\section{NUMERICAL EXAMPLE}

A numerical example will be presented in this section to demonstrate the practical use of the developed methodology. The example will consider a two-wheel mobile ground robot such as the one described in [23].

When considering the input as the voltage delivered to each of the motors and the output as the linear velocity $\left(y_{v}\right)$ and angular position $\left(y_{\theta}\right)$ of the robot, a model for the dynamics of the system can be obtained as ${ }^{2}$

$$
\begin{gathered}
P_{i}=\left(\left[\begin{array}{ccc}
0.878 & 0 & 0 \\
0 & 0.787 & 0 \\
0 & 0.089 & 1
\end{array}\right],\left[\begin{array}{ll}
0.066 & 0.061 \\
0.062 & -0.058 \\
0.003 & -0.003
\end{array}\right]\left[\begin{array}{cc}
1-f_{1} & \\
& 1-f_{2}
\end{array}\right],\right. \\
\left.\left[\begin{array}{ccc}
1-f_{3} & 0 & 0 \\
0 & 0 & 1-f_{4}
\end{array}\right], 0\right) .
\end{gathered}
$$

All fault models will be derived from (11), with each case represented by the parameters $f_{i}$ for $i \in \mathbb{N}_{[1,4]}$, the values of which are given in Table 1

\begin{tabular}{ccccc}
\hline$i$ & $f_{1}$ & $f_{2}$ & $f_{3}$ & $f_{4}$ \\
\hline 0 & 0 & 0 & 0 & 0 \\
1 & 0.334 & 0 & 0 & 0 \\
2 & 0 & 0.223 & 0 & 0 \\
3 & 0 & 0 & 0.399 & 0 \\
4 & 0 & 0 & 0 & 0.480 \\
\hline
\end{tabular}

TABLE I: Fault model parameters.

${ }^{2}$ For simplicity and brevity, the motors are given a static model (i.e.: modelled as a simple gain). This assumption does not affect the presented results and could be easily lifted if so desired. 
For this example, we impose the assumption that for all fault models $i, c\left(\boldsymbol{v}_{i}\right)=0$ and $c\left(\boldsymbol{w}_{i}\right)=0$. As a result of this assumption, we have that for all $\boldsymbol{v}_{i}$ and $\boldsymbol{w}_{i}$ there exist $v_{i} \in \mathbb{R}^{n_{x}}$ and $w_{i} \in \mathbb{R}^{n_{y}}$ such that $\boldsymbol{v}_{i}=\left[-v_{i}, v_{i}\right]$ and $\boldsymbol{w}_{i}=$ $\left[-w_{i}, w_{i}\right]$. Hence the model uncertainty can be quantified by the matrices

$$
\begin{aligned}
{\left[\begin{array}{lll}
v_{0} & \cdots & v_{4}
\end{array}\right] } & =10^{2} \cdot\left[\begin{array}{lllll}
1.629 & 1.827 & 0.557 & 1.930 & 1.914 \\
1.812 & 1.265 & 1.094 & 0.315 & 0.971 \\
0.254 & 0.195 & 1.915 & 1.941 & 1.601
\end{array}\right], \\
{\left[\begin{array}{lll}
w_{0} & \cdots & w_{4}
\end{array}\right] } & =10^{2} \cdot\left[\begin{array}{lllll}
5.250 & 3.766 & 7.099 & 12.462 & 8.245 \\
2.949 & 3.240 & 2.276 & 1.279 & 13.758
\end{array}\right] .
\end{aligned}
$$

Finally, we consider that all models have the same initial conditions, equivalent to the state at detection time.

For the simulation, the tracking error weight was set to $Q=10^{7} I$ thus enabling the tracking cost while $R$ was set to $R=I$. After 20 outer loop iterations (1.176 s) the algorithm terminated and the resulting separating input sequence was given by

$$
U_{[3]}=\left\{\left[\begin{array}{r}
-7.836 \\
0.659
\end{array}\right],\left[\begin{array}{r}
-7.629 \\
8.087
\end{array}\right],\left[\begin{array}{r}
-11.465 \\
12.000
\end{array}\right],\left[\begin{array}{r}
-11.642 \\
1.577
\end{array}\right]\right\},
$$

with $\Phi\left(U_{[3]}, 4\right)=6.589 \cdot 10^{-9}$ and $\Psi_{r}\left(U_{[3]}, 4\right)=5.401$. The results are shown in Figure 5. It can be observed that the generated input sequence achieves simultaneous output interval separation as desired.

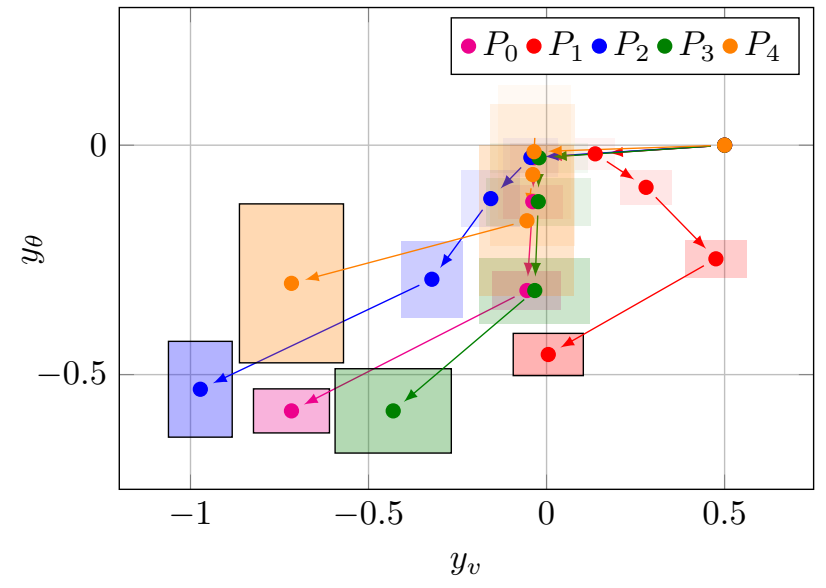

Fig. 5: Output trajectories under the separating input sequence $U_{[3]}$.

In the above example, note that in practice a maximum of 4 time steps is required for separation. By applying the computed sequence of control actions, we may be able to diagnose the fault in two steps if $P_{1}$ is the active model.

\section{CONCLUSION}

An active fault diagnosis methodology was presented in this paper based on set-membership methods. All possible system modes of operation (including the nominal and fault models) are modelled using interval arithmetic. The use of interval methods results in a more conservative approach but also gives easier to compute reachable sets and their pairwise distances. An optimization problem is formulated that outputs an input sequence that guarantees simultaneous model separation within some predefined horizon. The effectiveness of the developed algorithm is shown in simulation by an application to a mobile ground robot.

\section{REFERENCES}

[1] M. Blanke, M. Kinnaert, J. Lunze, M. Staroswiecki, and J. Schröder, Diagnosis and Fault-Tolerant Control. Springer-Verlag, New York, 2006.

[2] I. Punčochár and J. Skach, "A survey of active fault diagnosis methods," in 10th IFAC Symposium on Fault Detection, Supervision and Safety for Technical Processes, SAFEPROCESS 2018, 2018.

[3] H. Niemann, "A setup for active fault diagnosis," IEEE Transactions on Automatic Control, vol. 51, no. 9, pp. 1572-1578, sep 2006.

[4] A. E. Ashari, R. Nikoukhah, and S. L. Campbell, "Auxiliary signal design for robust active fault detection of linear discrete-time systems," Automatica, vol. 47, no. 9, pp. 1887-1895, 2011.

[5] N. Poulsen and H. Niemann, "Active fault diagnosis based on stochastic tests," International Journal of Applied Mathematics and Computer Science, vol. 18, no. 4, jan 2008.

[6] I. Punčochár, J. Siroky, and M. Šimandl, "Constrained active fault detection and control," IEEE Transactions on Automatic Control, vol. 60, no. 1, pp. 253-258, jan 2015.

[7] J. K. Scott, G. R. Marseglia, L. Magni, R. D. Braatz, and D. M. Raimondo, "A hybrid stochastic-deterministic input design method for active fault diagnosis," in 52nd IEEE Conference on Decision and Control. IEEE, dec 2013.

[8] G. Marseglia, J. Scott, L. Magni, R. Braatz, and D. Raimondo, "A hybrid stochastic-deterministic approach for active fault diagnosis using scenario optimization," IFAC Proceedings Volumes, vol. 47, no. 3, pp. 1102 - 1107, 2014, 19th IFAC World Congress.

[9] I. Punčochár and M. Šimandl, "On infinite horizon active fault diagnosis for a class of non-linear non-gaussian systems," Int. J Appl. Math. \& Comp. Sci., vol. 24, no. 4, pp. 795-807, dec 2014.

[10] D. M. Raimondo, R. D. Braatz, and J. K. Scott, "Active fault diagnosis using moving horizon input design," in 2013 European Control Conference (ECC). IEEE, jul 2013.

[11] L. Guibas, A. Nguyen, and L. Zhang, "Zonotopes as bounding volumes," in 14th ACM-SIAM Symp. on Discrete Algorithms, 2003.

[12] A. Ingimundarson, J. M. Bravo, V. Puig, T. Alamo, and P. Guerra, "Robust fault detection using zonotope-based set-membership consistency test," International Journal of Adaptive Control and Signal Processing, vol. 23, no. 4, pp. 311-330, apr 2009.

[13] J. K. Scott, R. Findeisen, R. D. Braatz, and D. M. Raimondo, "Input design for guaranteed fault diagnosis using zonotopes," Automatica, vol. 50, no. 6, pp. 1580-1589, jun 2014.

[14] J. K. Scott, D. M. Raimondo, G. R. Marseglia, and R. D. Braatz, "Constrained zonotopes: A new tool for set-based estimation and fault detection," Automatica, vol. 69, pp. 126-136, jul 2016.

[15] G. Marseglia and D. Raimondo, "Active fault diagnosis: A multiparametric approach," Automatica, vol. 79, pp. 223-230, 2017.

[16] F. Boem, A. Gallo, D. M. Raimondo, and T. Parisini, "Distributed fault-tolerant control of large-scale systems: an active fault diagnosis approach," IEEE Transactions on Control of Network Systems (Condtionally Accepted), 2018.

[17] A. Sathya, P. Sopasakis, A. Themelis, R. Van Parys, G. Pipeleers, and P. Patrinos, "Embedded nonlinear model predictive control for obstacle avoidance using PANOC," in European Control Conference, Limassol, Cyprus, Jun. 2018.

[18] L. Stella, A. Themelis, P. Sopasakis, and P. Patrinos, "A simple and efficient algorithm for nonlinear model predictive control," in 2017 IEEE 56th Annual Conference on Decision and Control (CDC), Dec 2017, pp. 1939-1944.

[19] B. Hermans, P. Patrinos, and G. Pipeleers, "A penalty method based approach for autonomous navigation using nonlinear model predictive control," in IFAC Conference on Nonlinear Model Predictive Control, Madison, USA, 2018.

[20] J. Nocedal and S. Wright, Numerical Optimization, 2nd ed., ser. Springer Series in Operations Research and Financial Engineering, T. V. Mikosch, S. I. Resnick, and S. M. Robinson, Eds. Springer.

[21] R. Rockafellar and R.-B. Wets, Variational Analysis, ser. Grundlehren der mathematischen Wissenshaften. Dordrecht: Springer, 2000, vol. 317.

[22] A. Kolmogorov and S. Fomin, Introductory real analysis, ser. Dover Books on Mathematics. New York: Dover Publications Inc., 1975.

[23] T. Urakubo, K. Tsuchiya, and K. Tsujita, "Motion control of a twowheeled mobile robot," Advanced Robotics, vol. 15, no. 7, pp. 711728, 2001. 